\title{
LOCKE Y LA ADULACIÓN ${ }^{*}$
}

\author{
Tomás Chuaqu H. \\ Instituto de Ciencia Política, Pontificia Universidad Católica de Chile
}

\begin{abstract}
Resumen
En este artículo se verifica la relevancia de un vicio político específico, la adulación, en las ideas de John Locke. Se muestra que para Locke la adulación proviene de agentes ilustrados que incitan a los aspirantes al poder recurriendo al orgullo que típicamente los caracteriza. Este vicio político, por ende, hace peligrar a los regímenes de poderes limitados y desconcentrados, ya que induce, a través del cultivo del orgullo, un deseo desmedido por el poder y el dominio sobre otros. Así, se precisa la importancia de la capacidad ciudadana para contener las pasiones y los vicios, propia además de la filosofía moral y de la educación de Locke, con vistas a la construcción y el mantenimiento de un régimen limitado, basado en el consentimiento, y protector de la vida libre.
\end{abstract}

Abstract

This article verifies the relevance of a specific political vice, namely, flattery, in John Locke's political thought. It is shown that for Locke flattery originates in learned agents whom incite aspirants to political power, appealing to the pride that typically characterizes them. This political vice, therefore, endangers the survival of political regimes of limited and decentralized powers since it incites, by catering to pride, an unchecked desire for power and domination over others. In this way, the importance of citizens' capacity to contain passions and vices, which pertains besides to Locke's moral and educational philosophies, is clarified in view of the construction and maintenance of a limited regime, based on the consent of the governed, and protective of freedom.

\section{PALABRAS CLAVE • Locke; • Adulación • Teoría Política • Liberalismo • Vicio Político}

\section{INTRODUCCIÓN}

No es común caracterizar a John Locke como un autor particularmente perspicaz respecto de las virtudes y vicios de lo político. Su obra es más reconocida por la estructuración conceptual de los fundamentos del buen gobierno, por la definición de las fronteras éticas del gobierno legítimo, o incluso por el diseño del ensamblaje institucional propio de un régimen adecuado a la modernidad. La teorización de lo político y de sus fines y medios a partir de una matriz de vicios y virtudes aparece como un ejercicio peculiarmente premoderno que Locke en efecto hubiera contribuido a desplazar ${ }^{1}$.

Una versión preliminar de este artículo fue presentada en el VII Congreso de la Asociación Chilena de Ciencia Política, el 26 de agosto del 2004, Universidad Central, Santiago de Chile.

Este punto es planteado en forma similar en Berkowitz, Peter. 1999. Virtue and the Making of Modern Liberalism. Princeton: Princeton University Press, pp. 74-76. 
En este artículo identifico un vicio político particular que aparece y reaparece en la obra de Locke y que percute en algunos de los argumentos centrales de su pensamiento político. Hasta donde he podido constatar, la presencia reiterada de este vicio en la obra de Locke no ha sido puntualizada anteriormente. Este vicio es la práctica de la adulación o la lisonja (flattery). Ensayando una definición general y sintética, que desglosaré más adelante, la adulación es el uso estratégico del elogio excesivo. Locke invoca esta categoría en momentos clave del desarrollo de sus argumentos políticos, es decir, aquéllos en los cuales identifica los mayores obstáculos y peligros para la construcción y el mantenimiento en existencia de un régimen de poderes limitados. En otras palabras, el objetivo de este trabajo es bien preciso y hasta modesto: demostrar que la capacidad para contener a la adulación ocupa un lugar relevante en el pensamiento político de Locke. Quisiera creer, eso sí, que tal objetivo involucra cuestiones más amplias ligadas a la naturaleza de un régimen de poderes limitados. Además, es posible que delinear la posición de la adulación en el contexto del pensamiento político de Locke contribuya a esclarecer algunas dificultades en la exégesis de su obra, como por ejemplo la relación entre sus reflexiones sobre la educación y la política, o el tipo de ciudadano necesario para ejercer la vida política libre.

\section{I.ADULACIÓN Y CONFIANZA}

La influencia de John Locke en el desarrollo del pensamiento político occidental es difícil de exagerar. Esto a pesar de que ya en 1969 uno de los más reconocidos intérpretes de su obra, John Dunn, se atrevía a decir: "I simply cannot conceive of constructing an analysis of any issue in contemporary political theory around the affirmation or negation of anything which Locke says about political matters"2. Dunn se retracta de tan osada afirmación unos veinte años después ${ }^{3}$, pero quizás alguna sospecha de que tal juicio contiene algo de verdad permanece. Originalmente Dunn creía que las premisas fundamentalmente religiosas del pensamiento de Locke impedían que sus ideas tuvieran resonancia en el presente. Dunn revisa esta posición sobre la base de un reconocimiento del grado en el cual Locke concibe lo político como una actividad esencialmente humana, y que por lo tanto su buen funcionamiento dependería de una frágil confluencia de voluntades humanas ${ }^{4}$. En el contexto de este argumento, Dunn releva la importancia de la categoría "trust", o "confianza", en el pensamiento de Locke. Es decir, el buen funcionamiento de lo político depende para Locke de la capacidad humana para confiar en la puesta en ejecución de la libertad de los demás ${ }^{5}$. Es claro que este es uno de los elementos que más

Dunn, John. 1969. The Political Thought of John Locke. Cambridge: Cambridge University Press.

Dunn, John. 1990. "What is living and what is dead in the political theory of John Locke?" En Interpreting Political Responsibility: Essays 1981-1989. Princeton: Princeton University Press.

$4 \quad$ Ibid., p. 13. "In particular what I failed completely to see [...] was just how vital and how deeply intelligent was Locke's understanding of the nature of politics as a purely human activity (an activity for humans for whom God has since gone off the air)."

5 "What trust fundamentally is is a device for coping with the freedom of other persons". Dunn, John. 1990. "Trust and Political Agency”, en Interpreting Political Responsibility: Essays 1981-1989, Princeton: Princeton University Press, p. 33. Para el origen de esta interpretación del sentido de la confianza, Dunn nos remite a la obra de Niklas Luhmann. 1979. Trust and Power, ed. T. Burns y G. Poggi. Chichester y New York: John Wiley.

Ver también, Dunn, John. 1984. "The Concept of Trust in the Politics of John Locke", pp. 279-301, en Philosophy in History: Essays on the Historiography of Philosophy, Richard Rorty, J.B. Schneewind, y Quentin Skinner, editores. Cambridge: Cambridge University Press.

Respecto de la categoría de "confianza" en general, véase la muy iluminadora serie de trabajos de Annette C. Baier: "Trust and Antitrust"; "Trust and its Vulnerabilities"; "Sustaining Trust"; y "Trusting People", todos incluidos en Moral Prejudices: Essays on Ethics, Cambridge: Harvard University Press, 1995, pp. 95-202. 
radicalmente distancian las ideas de Locke de las de Hobbes, a pesar de los variados intentos para sugerir su consanguinidad 6 . Para Hobbes no puede existir confianza mutua a menos que ésta sea forzada por el temor continuo y convergente en el Leviatán, mientras que para Locke, la confianza mutua es una premisa anterior a la existencia de la coacción gubernamental, y fundamenta éticamente la creación del gobierno. En alguna medida, esta diferencia explica el que Hobbes diseñe un gobierno total, mientras que Locke se esmere en demostrar que el único gobierno legítimo es uno cuyos poderes son restringidos y que está basado en el consentimiento ciudadano.

Si aceptamos el argumento de Dunn en cuanto a que el fundamento ético de la construcción del gobierno en Locke es una relación de confianza mutua existente entre los seres humanos, es lícito preguntarse qué es lo que hace peligrar la estabilidad y supervivencia de todo régimen basado en el consentimiento. El consentimiento individual conlleva el depósito de confianza en los demás que participan del acto que instaura el gobierno, y que se extiende y proyecta hacia la sociedad civil de la comunidad políticamente organizada. Pues bien: ¿quiénes son los que hacen peligrar el mantenimiento en existencia de un sistema político de poderes limitados y basado en el consentimiento como el que defiende Locke una vez que este está establecido? Locke designa una categoría de personajes en particular que se caracterizan por un vicio específico, la "flattery" ("adulación" o "lisonja"7), como especialmente nocivos para un régimen limitado. Los "flatterers" ("aduladores" o "lisonjeros") encienden el deseo desmedido por el poder, y por ende desestabilizan un régimen de poderes limitados. La adulación es un tipo de "abuso de confianza" en el que el adulador induce el engaño del adulado, atribuyéndole capacidades o virtudes de las que carece con el fin de ganar alguna ventaja; ;ocke les asigna a los aduladores un rol protagónico en provocar el deseo desmedido por el poder, lo que implica que se constituyen en uno de los obstáculos principales para la conservación de un sistema político basado en el consentimiento como el defendido en los dos Tratados.

En otras palabras, la adulación sería cierto tipo de vicio político que ocupa un lugar importante en las preocupaciones de Locke, y mi impresión es que ha sido pocas veces notado o remarcado el que en los argumentos de Locke se incluye la identificación de este vicio político, y que se lo asocia directamente con las amenazas que sobrevienen a la conservación de un régimen legítimo. En este sentido, tomo una perspectiva similar no sólo a la de John Dunn, como ya se ha visto, sino también a la que Peter Berkowitz asume en Virtue and the Making of Modern Liberalism ${ }^{9}$, aunque desde una óptica inversa, si se quiere: mientras Dunn y Berkowitz enfocan la vista en las virtudes políticas que estarían en la base del desarrollo del liberalismo, yo quisiera concentrar la atención en uno de los vicios políticos que debe ser evitado para proteger la existencia de un régimen

$6 \quad$ Ver, por ejemplo, Strauss, Leo. 1950. Natural Right and History. Chicago: The University of Chicago Press. Y, desde una perspectiva muy diferente, Macpherson, C. B. 1962. The Political Theory of Possessive Individualism: Hobbes to Locke. Oxford: Oxford University Press.

7 A través de este ensayo he optado en general por el uso del término "adulación" para traducir "flattery", ya que me ha parecido que las connotaciones políticas son más evidentes en este vocablo que en alternativas como "lisonja".

8 Esta relación entre la adulación y la confianza es notada ya en 1626 en la muy interesante pequeña obra de Edward Sutton The Serpent Anatomized: A Moral Discourse Wherein that FoulSerpentine Vice of Base Creeping Flattery is Manifestly Discovered and Justly Reproved. A needfull Caution for the Credulous, And very usefull for these Times. Kessinger Publishing's Rare Mystical Reprints, s.f. Dice Sutton: "for if thou meanest not to be deceived by him [el adulador], the best counsell that the wisest man can give thee, is not to trust him; for he that trusteth not, can hardly be deceived: and therefore, Though he speaketh favourably, beleeve him not." P. 2. La frase en cursivas proviene de Proverbios 26:25 y Sutton la repite solemnemente una y otra vez en el texto en forma bastante poco grácil, casi como un tosco leitmotiv.

9 Berkowitz, Peter. 1999. Virtue and the Making of Modern Liberalism. Princeton: Princeton University Press. 
limitado. Trataré de mostrar que la adulación constituye un elemento importante en la comprensión de los aspectos que Locke considera peligrosos para un régimen limitado. Esto podría contribuir a una mayor comprensión de los mecanismos disponibles para protegerlo.

Es bastante claro y sabido que una de las preocupaciones centrales de Locke es diseñar un régimen político de poderes limitados, en contraposición a la defensa de un régimen absoluto. Las obras políticas de Locke, en especial el Primer Tratado, pero también el Segundo, son escritos polémicos, que tienen como adversarios a aquéllos que defienden la derivación divina de la concentración del poder en un monarca. Para Locke, en cambio, un régimen legítimo es de poderes limitados y desconcentrados; en efecto, la concentración de poder es un riesgo permanente para todo gobierno legítimo. El peligro principal para Locke proviene de la tentación recurrente por concentrar excesivo poder en las manos de alguno o algunos. Para Locke es de gran importancia refutar los preceptos de sus adversarios absolutistas ya que para él, "there cannot be done a greater Mischief to Prince and People, than the Propagating wrong Notions concerning Government"10.

Recordemos, por cierto, que en el Primer Tratado del Gobierno Civil Locke señala en el subtítulo que los "False Principles and Foundation of Sir Robert Filmer, and His Followers, Are Detected and Overthrown"11. En su obra Patriarcha, Filmer había defendido un régimen absoluto derivado de la autoridad divina, en el entendido de que los reyes de la modernidad serían descendientes ni más ni menos que de Adán. Ahora, Locke insiste en que los argumentos de Filmer son sumamente débiles, y que ni siquiera sobreviven a los criterios más elementales del sentido común; de hecho en reiteradas ocasiones Locke sugiere que ni siquiera hay argumentos racionales en el texto de Filmer. Sin embargo, en uno de los ejemplos más egregios de "matar hormigas con bombas atómicas", como se dice comúnmente, en la serie de polémicas de la historia del pensamiento político, Locke le dedica páginas y páginas a la refutación de Filmer. ¿Por qué? Por un lado, Locke piensa que las ideas de Filmer resultaron muy influyentes -de aquí la referencia a los "seguidores" de Filmer- a pesar de su insustancialidad, y, por otro, considera que son especialmente insidiosas. Lo que me interesa aquí es determinar por qué Locke las considera tan insidiosas, dado que son, desde su punto de vista, patentemente insostenibles. Por supuesto, hay consideraciones de contexto histórico, en las que no voy a entrar, que podrían explicar el atractivo de los postulados de Filmer, pero también pienso que Locke está haciendo un punto más general con relación a los peligros que les sobrevienen a los regímenes limitados y legítimos.

Además, Locke está convencido de que aquéllos, como Filmer y sus seguidores, que difunden la falsa doctrina del derecho divino de los reyes a un poder absoluto la siembran en un terreno particularmente fértil, es decir, propicio para provocar un efecto pernicioso. ¿Cuál es este terreno?

Al referirse a Filmer y sus seguidores, Locke utiliza reiteradamente el calificativo al que ya he hecho referencia y que pienso es muy decidor. Hace alusión a los defensores del derecho divino de los reyes -es decir a aquellos que les atribuyen un poder absoluto a los monarcas- como "flatterers", o aduladores. De hecho, hasta donde he podido determinar, Locke utiliza el término en referencia a aquéllos que defienden esta doctrina en forma casi exclusiva. Es decir, pareciera tener un sentido bastante específico para él.

10

Locke, John. 1994. Two Treatises of Government, ed. Peter Laslett. Cambridge: Cambridge University Press, pp. 138. "Preface" al Primer Tratado. A través de este ensayo, las referencias a los Tratados se harán por número de página en esta edición, seguido de la sección correspondiente.

11 lbid., p.135. 
Por cierto, la adulación es una categoría de análisis muy antigua en la historia del pensamiento político, y no sólo del pensamiento político, sino más en general, del estudio de los caracteres humanos, y sin excepción, siempre con una connotación negativa y hasta ridícula. Es uno de los vicios más representados tanto en obras literarias y de filosofía moral, como también en la historia del pensamiento político. Tanto Platón como Aristóteles lo tratan, pero en general, con relación a los aduladores del demos, del pueblo, en el contexto del régimen democrático, en cuya circunstancia cae bajo la rúbrica del demagogo.

Basándome en parte en el muy perspicaz análisis de Jean Starobinski ${ }^{12}$ describiré, para comenzar, la dialéctica de la adulación, cuya característica esencial es el elogio excesivo, o la expresión exagerada de admiración, con fines estratégicos. Es decir, el adulador persigue congraciarse con el adulado, de tal manera de conseguir algún beneficio.

Tal como apunta Starobinski, uno de los lugares más clásicos de referencia a los aduladores es la obra Caracteres ${ }^{13}$ de Teofrasto, el sucesor de Aristóteles a la cabeza del Liceo. Teofrasto, a pesar de que describe al adulador en el contexto de alguien que adula a un particular, y no de alguien que está en una posición de poder político, ya establece uno de los elementos más significativos de esta actividad: el adulador elogia excesivamente a alguien con vistas a la consecución de un beneficio propio, y es claro que se trata de un personaje ridículo, digno de desprecio. Teofrasto nos infiere también que la adulación se remite a alguien que está en una posición para distribuir beneficios, ya sea materiales o de poder, y que la motivación del lisonjero es avanzar su interés propio: el lisonjero se hace ridículo para conseguir un beneficio: "Se podría definir la adulación como un trato indigno [en sí mismo,] pero ventajoso para quien lo práctica"14. El adulado, sin embargo, el objeto de la adulación, no es caracterizado por Teofrasto, quien deja en la nebulosa el carácter de quien se deja adular.

Mucho tiempo después, sin embargo, es Shakespeare, en el Timón de Atenas ${ }^{15}$, quien consagra al adulado. No será esta la más remarcable de las obras de Shakespeare -de hecho se trata de un esfuerzo conjunto con Thomas Middleton ${ }^{16}$, autor, claro, de talento menos notable que el de Shakespeare- pero en ella la adulación, y sus muy dañinos efectos sobre el adulado, se configuran en el centro de la acción dramática. Obviamente, la adulación es encarnada por algunos de los villanos más memorables de Shakespeare: baste recordar, por ejemplo, a Regan y Goneril en King Lear, a lago en Othello, o al mismísimo Ricardo III (especialmente cuando aún es Duque de Gloucester). Cabe hacer notar que la temática relativa a la adulación adquiere una relevancia especial en la historia de las ideas cuando se comienza a desarrollar la reflexión sobre la vida en las cortes reales, especialmente durante el Renacimiento, y en este período es esencial la referencia a II Cortegiano de Castiglione, y por supuesto, al capítulo XXIII, "De cómo hay que huir de los aduladores" de El Príncipe de Maquiavelo, ambas obras bien conocidas por Shakespeare, que

"De la lisonja", Capítulo II de Remedio en el mal: Crítica y legitimación del artificio en la era de las luces, A. Machado Libros, S.A., 2000 (versión original en francés, Éditions Gallimard, 1989), pp. 15-105. Para una revisión más completa, aunque ligera y juguetona, de la historia de la adulación, ver Stengel, Richard. 2000. You're too Kind: A Brief History of Flattery. New York: Touchstone.

13 "De la adulación", en Teofrasto, Caracteres, Madrid: Editorial Gredos, 2000, pp. 50-52. Ibid., p. 50. Paréntesis cuadrados en original.

Shakespeare, William y Middleton, Thomas. 2004. The Life of Timon of Athens. Oxford: Oxford University Press.

Ver John Jowett, "Introduction" a Shakespeare y Middleton 2004, especialmente pp. 132-153. 
desde perspectivas distintas pretenden regular el comportamiento apropiado de los que rodean a los mandatarios. "Como regla general, [la adulación] tiene su campo de acción dondequiera que un poderoso, un rico, un superior tiene 'corte', lugar de acogida de parásitos o clientes"17. Además, no debiera sorprender que la adulación sea un topos en Shakespeare, ya que tantas de sus obras tratan de las intrigas de la vida cortesana ${ }^{18}$.

Pero en Timón ocupa un lugar preeminente por cuanto es el detonante del desarrollo de la tragedia del personaje principal. Timón es un pudiente noble de Atenas que peca de excesiva generosidad ante los que se hacen pasar por sus admiradores y que vierten elogios sobre su persona con un desenfado incomparable. El trágico destino de Timón -quien termina en la bancarrota luego de que sus falsos amigos le dan la espalda cuando sus recursos se acaban, habiéndolos despilfarrado en regalos a los lisonjeros que lo han rodeado- se deriva de su excesiva ingenuidad, que le impide reconocer la falsedad de sus "admiradores". En este sentido, Shakespeare nos ofrece una psicología del adulado -que quizás también aparece en Otelo o Lear- que Teofrasto no había ofrecido, y que se resume en la frase de Apemanto, el rudo filósofo que es el único amigo desinteresado de Timón y que se atreve a decirle las cosas como son: "he that loves to be flattered is worthy o' the flatterer"19. Es decir, el adulador y el adulado son igualmente despreciables y reprochables: son cómplices en el vicio.

Aquí ya aparece uno de los temas centrales de la reflexión relativa a la adulación: el adulado no es sencillamente una víctima inocente del engaño del adulador. Timón, por ejemplo, suscita su propia desventura al dejarse llevar por una ingenuidad que linda con la frivolidad: los aduladores que lo rodean se aprovechan de su incapacidad para reconocer la zalamería que practican para enriquecerse a su costa. Es interesante hacer notar que al reconocer finalmente la falsedad de sus compatriotas, Timón se convierte en un misántropo, esto es, en alguien que ha perdido completamente la capacidad de confiar en los seres humanos, ya que los considera naturalmente corruptos. Es decir, el exceso de confianza del que Timón padece originalmente permite que se abuse de él, causando el efecto contrario, es decir, la ausencia total de confianza en los demás: "[...] for the unthankfulness of those he had done good unto and whom he took to be his friends, he was angry with all men and would trust no man"20. Sin embargo, el punto esencial en la lógica del Timón,

Además de las obras ya mencionadas, también podrían incluirse en la lista Pericles y Julio César. Sólo como ejemplo, cito estos notables versos de Decius, uno de los conspiradores en contra de Julio César:

"for he loves to hear

That unicorns may be betray'd with trees,

And bears with glasses, elephants with holes,

Lions with toils, and men with flatterers;

But when I tell him he hates flatterers,

He says he does, being then most flattered."

(Julius Caesar, ed. T. S. Dorsch, Londres: The Arden Shakespeare, 1977, p. 44. Act II, Sc. 1, 203-208.)

19 Timon of Athens, op. cit., Act I, Sc. 1, 229-230, p.185. Es interesante constatar el parentesco dramático entre Apemanto y el Fool de Lear. El bufón y el filósofo están en una posición como para darse el lujo de no verse obligados a practicar la adulación, al estar cerca del poder, pero no dependen enteramente de él, y su función es decir la verdad, aunque sea en enigmas que sólo los entendidos descifran.

20 Ibid., Appendix B: From Plutarch's 'Life of Marcus Antonius', translated by Thomas North, p. 331. La traducción de Thomas North de las Vidas Paralelas es una de las fuentes principales del Timón, como también de otras obras de Shakespeare basadas en personajes griegos o romanos, como Coriolano o Julio César. 
como en todas las tragedias de Shakespeare, es que es una debilidad de carácter propia del personaje principal la que causa su trágico destino. En el caso de Timón, su vulnerabilidad a la lisonja se debe a su vanidad.

Esta inflexión en la caracterización de la adulación es remarcada por La Rochefoucauld. Tomemos, por ejemplo, las máximas 2 y 152: "El mayor lisonjeador de todos es el amor propio"; "Si no nos halagáramos no podría sernos nociva la lisonja de los demás"; y la 158: "La lisonja es falsa moneda que sólo por nuestra vanidad halla curso"21. Lo interesante de esta versión de la lisonja es que su campo de acción ya no es sólo la ingenuidad, o la carencia de sagacidad para reconocer a quienes nos engañan con vistas a su beneficio. El campo de acción de la lisonja es un vicio bien particular: es el amor propio, la vanidad o el orgullo del adulado. Por ende, la adulación tiene efecto sólo donde encuentra un campo de acción, es decir, un orgullo suficientemente susceptible a la lisonja, que bloquea el reconocimiento del ridículo que hace el adulador.

Esto proporciona al adulador un gran espacio abierto en medio de la amistad, al tener como una útil base de operaciones contra nosotros nuestro amor por nosotros mismos, por el que, siendo uno mismo, el principal y más grande adulador de sí mismo, admite sin dificultad al de fuera como testigo, y como autoridad aliada garante de las cosas que piensa y desea22.

De hecho, el adulador, para ser eficaz en sus propósitos, debe jugar un doble juego: debe elogiar en exceso, esforzándose a la vez por no ser descubierto, tanto en cuanto a sus motivaciones, como en cuanto a lo hiperbólico de sus halagos. Dejar en evidencia al adulador como tal, socava sus proyectos.

\section{II.LA ADULACIÓN EN EL PRIMER TRATADO Y EN SOME THOUGHTS CONCERNING EDUCATION}

¿Qué tiene que ver todo esto con Locke? Volvamos a la pregunta inicial, esto es, ¿qué o quiénes son particularmente peligrosos para la conservación de un régimen limitado? Por cierto, la respuesta más sencilla es "aquéllos que aspiran a hacerse de un poder desmedido", es decir, en el contexto de Locke, los que aspiran a convertirse en monarcas absolutos. Sin embargo, en los dos tratados, Locke no ataca directamente a los aspirantes a la monarquía absoluta, 0 a aquellos que la reclaman para sí mismos. Sus dardos van dirigidos especialmente contra quienes adulan, 0 hacen uso de la adulación, para suscitar el deseo, o la pasión por el poder absoluto, y más en general, el prurito por ejercer dominio sobre los demás.

Dado que, según Locke, la ley natural y la razón suscitan la instauración de un régimen dedicado a la protección de la vida, la libertad y la propiedad, sólo el recurso a lo pasional puede explicar la pertinacia de ideas erróneas y perniciosas sobre el gobierno. Todos somos susceptibles a dejarnos llevar por las pasiones, pero estas pueden ser suscitadas e impulsadas a través de la adulación: Locke querría ponerles un freno a las pasiones, especialmente a aquéllas que hacen peligrar al gobierno legítimo, a través de dos mecanismos interconectados entre sí con relación a la

21 La metáfora de la moneda falsa parece originarse, como en tantas otras figuras relativas a la adulación, en Plutarco: ver, "Cómo distinguir a un adulador de un amigo" en Plutarco. 1985. Obras morales y de costumbres (Moralia), Madrid: Editorial Gredos p. 242.

22 Ibid., p. 201. 
adulación: poner al descubierto a los aduladores, exponiéndolos al ridículo; y proveyendo argumentos racionales alternativos. En términos esquemáticos se podría decir que la ironía y el desprecio que el normalmente flemático Locke manifiesta en el Primer Tratado respecto de Filmer y sus seguidores cumplirían el primero de estos objetivos; y el intento por derivar racionalmente un régimen alternativo en el Segundo Tratado, el segundo.

Dice Locke, cerca del comienzo del Primer Tratado: "In this last age a generation of men has sprung up among us, who would flatter princes with an Opinion, that they have a Divine Right to absolute Power"23. Esta adulación es sumamente peligrosa justamente porque se infiltra en la vanidad natural de aquellos que se inclinan hacia el poder. Para Locke la posición de Filmer es tan osada que hubiera requerido una batería sumamente potente de argumentos para su aceptación. Sin embargo, cree Locke que la potencia de la posición de Filmer no se deriva de su argumentación, que como he dicho Locke considera una sarta de falacias, sino más bien del campo de acción en el que se ejecuta la adulación:

Our A- [Filmer] having placed such a mighty Power in Adam, and upon that supposition, founded all Government, and all Power of Princes, it is reasonable to expect, that he should have proved this with Arguments clear and evident, suitable to the weightiness of the Cause. That since Men had nothing else left them, they might in Slavery have such undeniable Proofs of its Necessity, that their Consciences might be convinced, and oblige them to submit peaceably to that Absolute Dominion, which their Governors had a right to exercise over them. Without this, What Good could our A- do, or pretend to do, by erecting such an unlimited Power, but Flatter the Natural Vanity and Ambition of Men, too apt of it self to grow and encrease with the Possession of any Power? And by perswading those, who, by the consent of their Fellow-Men, are advanced to great, but limited degrees of it, that by that part which is given them, they have a Right to all, that was not so; and therefore may do what they please, because they have Authority to do more then others, and so tempt them to do what is neither for their own, nor the good of those under their Care, whereby great Mischiefs cannot but follow24.

En efecto, lo que produce persuasión, no es, por lo tanto, la fuerza de los argumentos de Filmer y sus seguidores, sino el interés tanto de aquellos que se harían del poder absoluto, como de aquellos que persiguen beneficiarse de tal poder, a través de la consecución de cuotas de poder particular, o posiblemente de dádivas materiales. Locke desafía a los seguidores de Filmer -ya muerto, claro, cuando Locke escribe- a que localicen en Patriarcha argumentos que de alguna manera sustenten sus aventuradas propuestas:

If no such Arguments are to be found, I beseech those Men, who have so much cryed up this Book, to consider whether they do not give the World cause to suspect, that it's not the Force of Reason and Argument, that makes them for Absolute Monarchy, but some other by Interest, and therefore are resolved to applaud any Author, that writes in favour of this Doctrine, whether he support it with Reason or no ${ }^{25}$.

Tal como dice Starobinski, "Entre el adulador y el tirano la apuesta es a un tiempo más alta y más peligrosa: lo que está en juego es el poder mismo, y el favor se traduce en inmensas riquezas. La

Locke, op. cit., p. 142. 1er T, sección 3.

Ibid., p. 148. 1er T, sección 10.

Ibid., p. 150. 1er T, sección 13. 
lisonja da ahí libre curso a su figura favorita, la hipérbole. Diviniza al príncipe; le concede la satisfacción de todo deseo; remueve todo obstáculo que intentara oponer la virtud"26. Justamente, Locke acusa a Filmer y sus seguidores de "divinizar" al monarca, e hinchar su ambición en forma estratégica para obtener beneficios de él.

Ahora bien, esto sería poco más que un argumento ad hominem si Locke no se diera el tedioso trabajo de ensayar la refutación, una a una, de las sugerencias de Filmer, cosa que explica la cantidad de tinta que Locke expende en el Primer Tratado. Pero el punto aquí es que Locke ya en el Primer Tratado utiliza la dialéctica de la adulación tal como es representada por La Rochefoucauld y otros -es decir, el juego entre la adulación, el orgullo, y el interés propio-para explicar el atractivo de la defensa del poder absoluto de los reyes. En otras palabras, existe en el Primer Tratado una psicología implícita del vicio político que, veremos, es desarrollada en el Segundo Tratado.

Siguiendo como decía la sugerencia de Berkowitz, aunque desde una óptica bastante distinta, quiero argumentar que para Locke la creación de un gobierno civil como el que propone requiere, si no como argumenta Berkowitz la virtud, sí la capacidad para contener cierto vicio. Esto es la capacidad para reprimir el deseo de dominio sobre el resto. En la psicología moral de Locke, uno de los elementos más significativos es justamente la capacidad para mantener los deseos bajo control.

Esta línea de razonamiento se expresa en forma especialmente clara en Some Thoughts Concerning Education. En efecto, el objetivo de las reflexiones sobre la educación es proponer, en forma no demasiado sistemática, estrategias pedagógicas para impulsar el desarrollo del sentido moral en las personas, y, tal como dicen Yolton y Yolton en su Introducción, esta obra es sumamente relevante al pensamiento político de Locke: "The links between Some Thoughts and Two Treatises are especially important; the former provides a training and educational programme for the development of a moral person, the latter places that person in the political arena"27. Las ocasiones en las que Locke hace referencia a la adulación en Some Thoughts ameritan alguna consideración en este sentido.

Locke es generalmente considerado como un teórico progresista en cuanto a la educación de los niños, sin desconocer que sus ideas están dirigidas en especial a la buena crianza de un "gentleman" de la clase pudiente, o como dice Locke, "suited to our English Gentry"28. Por ejemplo, en el contexto de su discusión en cuanto al uso relativo de castigos y recompensas con la intención de inducir el buen comportamiento o el aprendizaje de los niños, Locke argumenta que el elogio excesivo y el uso indiscriminado de recompensas materiales son nocivos por cuanto incapacitan a los niños para controlar sus propios impulsos.

Beating them, and all Sorts of slavish and corporal Punishments, are not the discipline fit to be used in the Education of those we would have wise, good, and ingenuous Men; and therefore

Satrobinski, op. cit., p. 85.

John Locke. 2003. Some Thoughs Concerning Education, John W. y Jean S. Yolton, Editores, Oxford: Oxford University Press, p. 1. Un punto similar es desarrollado por Christopher Anderson en “'Safe enough in his honesty and prudence:' The ordinary conduct of government in the thought of John Locke", History of Political Thought, Vol. XIII, N ${ }^{\circ} 4$, Winter 1992, pp. 605-630.

28 Some Thoughts, op. cit., p. 80. Por supuesto, es justamente de esta clase de donde provienen en su mayoría aquéllos que accederían a posiciones de liderazgo político y cultural, tal como hace ver Anderson, op. cit., p. 613. No obstante, Anderson se apura en asegurar que Locke consideraba que todos los hombres nacen con una capacidad innata para el pensamiento racional. Op. cit., p. 614 . 
very rarely to be applied, and that only in great Occasions, and Cases of extremity. On the other side, to flatter Children by Rewards of things, that are pleasant to them, is as carefully to be avoided. He that will give his Son Apples, or Sugar-plumbs, or what else, of this kind, he is most delighted with, to make him learn his Book, does but authorize his love of pleasure, and cocker up that dangerous propensity, which he ought by all means to subdue and stifle in him. [...] Thus People, to prevail with Children to be industrious about their Grammar, Dancing, or some other such matter, of no great moment to the happiness or usefulness of their Lives, by misapplied Rewards and Punishments sacrifice their Vertue, invert the Order of their Education, and teach them Luxury, Pride, or Covetousness, etc. For in this way, flattering those wrong Inclinations, which they should restrain and suppress, they lay the Foundations of those future Vices, which cannot be avoided, but by curbing our Desires, and accustoming them early to submit to Reason ${ }^{29}$.

Lo interesante de este pasaje es que Locke muestra cómo la adulación, en este caso en una versión materializada en golosinas que placen a los niños, induce vicios -y nótese que se incluye el orgullo entre ellos- y promueve la incontinencia. Es en este mismo sentido que Locke, a pesar de que no es partidario de la aplicación de castigo físico, recomienda cierto grado de firmeza en la reprimenda cuando ésta corresponda. En efecto, más que recomendar la reiteración de la reprimenda, Locke sugiere que es más efectivo hacerles ver y sentir a los niños que mientras no corrijan su comportamiento, o se disculpen por su mala acción, están en un estado de desgracia, constatable por el disgusto que sus mayores manifiestan en su compañía. Lamentablemente, en el hogar de un gentleman este esfuerzo enfrenta algunas dificultades, derivadas de la interferencia de la servidumbre en la educación de los niños.

The great Difficulty here, is, I imagine, from the Folly and Perverseness of Servants, who are hardly to be hinder'd herein the Design of the Father and Mother. Children, discountenanced by their Parents for any Fault, find usually a Refuge and Relief in the Caresses of those foolish Flatterers, who thereby undo whatever the Parents endeavour to establish ${ }^{30}$.

Nótese que el lenguaje de este pasaje es sorprendentemente duro -"Folly, Perverseness, foolish"-, remarcando la importancia que Locke le asigna al daño que este tipo de adulación produce. Y no es para menos, ya que como hemos visto, induce algunos de los peores vicios. Creo que es de notar que estos pasajes sobre la educación de los niños tienen resonancias evidentemente políticas: todos aquéllos que hemos tenido la mala fortuna de sufrir la presencia de niños mal enseñados sabemos que es común que se comporten como pequeños tiranos, demandando la satisfacción de todos y cada uno de sus deseos y apetitos sin importarles los sacrificios de los que los rodean. En otras palabras, la adulación en la educación de los niños gesta hombres ${ }^{31}$ de apetitos desmedidos, incapacitados para hacerse parte de una comunidad política regida por un gobierno de poderes limitados

Some Thoughts, op. cit., pp. 113-114.

Ibid., p. 117.

Uso el lenguaje masculino para reflejar adecuadamente el de Locke, y no mi propio pensamiento. Para una interesante y reciente discusión sobre las ideas de Locke respecto a los derechos de las mujeres, ver Jeremy Waldron, 2002, God, Locke and Equality: Christian Foundations in Locke's Political Thought, Cambridge: Cambridge University Press, en especial el Capítulo 2, "Adam and Eve", pp. 21-43. Waldron ofrece una visión muy equilibrada del asunto, y es particularmente útil la discusión que ofrece sobre las muy inteligentes e influyentes ideas de Carole Pateman 1988, en, The Sexual Contract, Stanford: Stranford University Press. 
y basado en la igualdad ciudadana. El autogobierno de los apetitos está íntimamente ligado al autogobierno de un pueblo soberano, por cuanto este tipo de régimen requiere ciudadanos capaces de restringir sus deseos y controlar sus pasiones, especialmente aquéllas, como el orgullo, que incitan al dominio sobre otros. Este aspecto del carácter moral de los ciudadanos, por cierto, es especialmente relevante en aquéllos que pudieran estar en una posición de influencia y poder, como los miembros de la gentry, quienes por estar en una posición privilegiada para recibir una educación que desarrolle su racionalidad, tienen el deber de hacer uso de sus recursos materiales y del ocio del que gozan para una formación que potencie sus cualidades morales.

Si la capacidad para no ser vulnerable a los perversos efectos de la adulación es central en la propuesta educativa de Locke, también lo es la capacidad para evitar su práctica.

He that knows how to make those he converses with easie without debasing himself to low and servile flattery, has found the true art of living in the World, and being both welcome and valued every where. Civility therefore is what in the first place should with great care be made habitual to Children and Young people. [...]

The thing they should endeavour and aim at in Conversation, should be to shew Respect, Esteem, and Good-will, by paying to every one that common Ceremony and Regard which is in civility due to them. To do this, without suspicion of Flattery, Dissimulation, or Meanness, is a great Skill, which good Sense, Reason, and good Company can only teach; but is of so much use in civil Life, that it is well worth the studying ${ }^{32}$.

Es importante notar que la adulación es propia de los serviles, y no de aquellos que tratan a los demás con la consideración y respeto que merecen. Quizás por esta razón Locke sugiere que los sirvientes son especialmente proclives a su uso. Su posición subordinada los induce a adular, probablemente como un mecanismo para convertir al niño en un pequeño tirano de sus padres, de tal manera de avanzar sus propios intereses. Pero, si la adulación es reprochable en los sirvientes -aunque posiblemente previsible dada su circunstancia servil- es sencillamente inadmisible en un gentleman, quien debe estar preparado para hacerse párte de la vida civil y relacionarse, diríamos, en forma civilizada con quienes lo rodean. La adulación es antisocial y propia de la vida ruda, insensata, e irracional, acorde al imperio de las pasiones y de los vicios, acorde, en fin, a aquéllos destinados a la esclavitud de los que viven bajo un tirano.

Ahora, no obstante que la adulación es propia de los serviles, esto no significa que sea un vicio especialmente peligroso de la gente común. La adulación es una habilidad perversa característica de personas con altos niveles de preparación intelectual, como Filmer y sus seguidores. Ya hemos notado la gravedad que Locke le atribuye a la propagación de ideas erradas sobre el gobierno, y éstas ciertamente son difundidas por miembros de una elite instruida y sofisticada. Jeremy Waldron, en el contexto de una defensa del igualitarismo fundamental de la filosofía social de Locke, argumenta de manera similar:

It is in many ways the educated intellect that Locke regarded as a social danger. He often said that the capacities and dispositions of ordinary people were much more reliable morally and politically than the effete corrupt sensibility of "all-knowing Doctors" and "learned Disputants". When he cited those who had undermined the basis of trustworthy government in England, it 
was learned men-scholars, statesmen, bishops, "the Divinity of this last Age," and "Flatterers [who] talk to amuze Peoples Understandings." There is no suggestion of any threat from masterless men or from halting attempts at political thinking by uppity laborers. On the contrary, when Locke considers lower-class malcontents, he is anxious to assure his readers that they are not a real threat at all, certainly not compared to those who are in a position to flatter and encourage the pretensions of absolute power ${ }^{33}$.

En otras palabras, son los letrados quienes violentan al buen gobierno, pues son los que tienen más que ganar y menos que perder con la instauración del poder absoluto. Justamente es en el Segundo Tratado, dedicado a la defensa de un régimen limitado y de la soberanía popular, donde Locke más claramente precisa el daño que los aduladores causan.

\section{III.LA ADULACIÓN EN EL SEGUNDO TRATADO}

La adulación aparece en el Segundo Tratado en tres momentos clave de su argumentación: 1) con relación a la posible constitución histórica de un régimen de poderes limitados; 2) en la discusión sobre los derechos de prerrogativa del poder ejecutivo; y 3) en la larga reflexión sobre la disolución de los gobiernos. En otras palabras, Locke introduce la categoría en aquellos pasajes en los que discute el origen de un régimen legítimo, los peligros para su mantenimiento en existencia, y su final disolución.

En cuanto a los orígenes del gobierno, Locke argumenta que la fuente de la discordia en el estado natural, regido como es bien sabido por la ley natural, es el hecho de que en esta circunstancia cada uno es juez en su propia causa, lo que dificulta la posibilidad de llegar a acuerdos justos cada vez que se da una controversia, puesto que somos proclives a la parcialidad y a darles preferencia a nuestros propios intereses. El estado natural es, por lo tanto, pacífico, ya que se rige por la ley natural, que los hombres son capaces de obedecer; pero incierto, justamente porque en él la paz depende de esta falible capacidad humana ${ }^{34}$. Por ende, para hacerse parte del pacto que configura a la sociedad civil habría que estar dispuesto a ceder la facultad del juicio a una autoridad común, basada en el consentimiento. En efecto, un monarca absoluto, según Locke, estaría en una relación de estado natural con todo el resto de la sociedad en tanto sería juez en su propia causa: de hecho, la sumisión a un monarca absoluto es peor que el estado natural, por cuanto el monarca absoluto está "corrompido por la adulación" y además "armado de poder": "corrupted with Flattery and armed with Power" 35 .

Ahora bien, a pesar de que Locke considera que los gobernantes absolutos son ilegítimos, reconoce que históricamente es posible que las primeras sociedades se hayan configurado alrededor del poder de aquél considerado el más virtuoso en pos de la consecución del bien común; Locke invoca en la sección 111 el mito de la edad de oro con este propósito, pero argumenta que:

Waldron, op. cit., p. 85. Las referencias a la obra de Locke en la cita corresponden a: An Essay Concerning Human Understanding, ed. P. H. Nidditch, Oxford: Clarendon Press, p. 495, y a las secciones 94, 112 y 230 del Segundo Tratado.

34 Para una exposición especialmente lúcida, y de la que me he beneficiado mucho en esta sección, del uso del modelo del estado natural en Locke, ver Richard Ashcraft, "Locke's State of Nature: Historical Fact or Moral Fiction?", en The American Political Science Review, Vol. 62, № 3 (Sept. 1968), pp. 898-915.

35 Locke, Two Treatises, p. 327, $2^{\circ}$ T sección 91. 
when Ambition and Luxury, in future Ages would retain and increase the Power, without doing the Business, for which it was given, and aided by Flattery, taught Princes to have distinct and separate Interests from their People, Men found it necessary to examine more carefully the Original and Rights of Government; and to find out ways to restrain the Exorbitancies, and Prevent the Abuses of that Power which they having intrusted in another's hands only for their own good, they found was made use of to hurt them ${ }^{36}$.

En este muy rico pasaje sólo quiero hacer notar la función que Locke le atribuye a la adulación: induce un vicio político de parte de los que poseen el poder, esto es, el desligar su interés propio del interés de la comunidad, y por ende genera el abuso del poder irrestricto. La edad de oro sería algo así como una segunda etapa en el desarrollo de la historia humana, en la que la ley natural aún se respeta, a pesar de que los hombres se organizan bajo el mando de uno, el más virtuoso ${ }^{37}$. Este tipo de organización introduce la vulnerabilidad del sistema a la adulación. Ya en sus orígenes históricos, entonces, la sociedad legítima resulta del intento por controlar los abusos del poder derivados, al menos en parte, de los efectos nocivos de la adulación.

Más adelante, al analizar el derecho de prerrogativa del poder ejecutivo, Locke igualmente atribuye su abuso original, al menos en parte, a la acción de la adulación, y concibe el origen del gobierno limitado como resultado del reconocimiento de parte del pueblo de los abusos de la prerrogativa de los príncipes, resultantes de la adulación que imperaría sobre príncipes débiles. Nótese que Locke conecta la susceptibilidad a la adulación con la debilidad, que creo entender no tanto como debilidad de poder, sino como debilidad moral, en el sentido de dejarse llevar por la persecución de intereses propios por sobre los intereses del pueblo:

It is easie to conceive, that in the Infancy of Governments [...] the Government was almost all Prerogative. A few establish'd Laws served the turn, and the discretion and care of the Ruler supply'd the rest. But when mistake, or flattery prevailed with weak Princes to make use of this Power, for private ends of their own, and not for the publick good, the People were fain by express Laws to get Prerogative determin'd, in those points, wherein they found disadvantage from it: And thus declared the limitations of Prerogative were by the People found necessary in Cases, which they and their Ancestors had left, in the utmost latitude, to the Wisdom of those Princes, who made no other but a right use of it, that is, for the good of their people ${ }^{38}$.

Por lo tanto, es también la adulación la que induce a que el uso legítimo de la prerrogativa del ejecutivo -que "no es otra cosa que el poder de hacer un bien público sin regla alguna"; "nothing but the Power of doing publick good without a Rule"39- sea explotado en el gobierno basado en el consentimiento. El desarrollo del Estado de derecho basado en leyes explícitamente estipuladas y cuyo objeto esencial es restringir la autoridad pública a espacios de poder determinados fue una reacción al uso perverso de la prerrogativa que los aduladores impulsan.

De hecho, son los buenos príncipes quienes más hacen peligrar la vida libre, por cuanto son capaces, dada su virtud, de hacer uso de sus prerrogativas eficazmente para avanzar el bien

Ibid., p. 343, 2 T sección 111.

Ver Ashcraft, op. cit., pp. 908-914 para este argumento.

Locke, Two Treatises, op. cit., p. 376, $2^{\circ}$ T sección 162.

Ibid., p. 378. $2^{\circ} \mathrm{T}$ sección 166. Cursivas en el original. 
común: "Upon this is founded that saying, That the Reigns of good Princes have been always most dangerous to the Liberties of their People" ${ }^{40}$. Los sucesores de tales príncipes excepcionales -que lo son tanto que incluso son "God-like"41 _ serían necesariamente incapaces de continuar tal eficacia. Así, los mejores príncipes dañan a la comunidad política, estableciendo prácticas de gobierno no reguladas y arbitrarias. Por lo tanto, creo que se puede decir con cierta seguridad que son, en parte, los nocivos efectos de la adulación los que provocan la necesidad de generar un gobierno limitado, y continúa siendo un peligro incluso luego de la instalación de un gobierno legítimo. De hecho, es en el contexto de una asociación civil donde la adulación genera sus más perniciosos efectos sobre la debilidad moral de los que sienten un prurito por el poder.

Cuando Locke arriba a la discusión relativa a la disolución de los gobiernos legítimos, en el último capítulo del Segundo Tratado, la categoría de adulación vuelve a aparecer, y ahora, reiteradamente (tres usos literales del término), y ligada en forma expresa al orgullo y la vanidad. Posiblemente sea en estos pasajes donde ocupa el lugar más importante. Típicamente para Locke la disolución de un gobierno legítimo se da cuando el príncipe abusa de su poder ${ }^{42}$.

Why in such a Constitution as this [la defendida en el $2^{\circ} \mathrm{T}$, the Dissolution of the Government in these Cases is to be imputed to the Prince, is evident: because he having the Force, Treasure, and Offices of the State to imploy, and often perswading himself, or being flattered by others, that as Supream Magistrate he is uncapable of controul; he alone is in a Condition to make great Advances toward such Changes, under the pretence of lawful Authority, and has it in his hands to terrifie or supress Opposers, as Factious, Seditious, and Enemies to the Government: Whereas no other part of the Legislative, or People is capable by themselves to attempt any alteration of the Legislative, without open and visible Rebellion, apt enough to be taken notice of; which when it prevails, produces Effects very little different from Foreign Conquest ${ }^{43}$.

Es decir, los príncipes están en una situación particularmente vulnerable al efecto de la adulación, y esta vulnerabilidad es un peligro permanente de la existencia del poder ejecutivo centrado en el príncipe.

Aquellos, como Filmer y sus seguidores, que propugnan el derecho divino de los reyes, devienen en defensores de la disolución del gobierno legítimo en el estado de guerra, que no es más que equivalente al estado de sometimiento al arbitrio no regulado por leyes de otro.

[T]hose who set up force again in opposition to the Laws, do Rebellare, that is, bring back again the state of War, and are properly Rebels: Which they who are in Power (by the pretence they have to Authority, the temptation of force they have in their hands, and the Flattery of those about them) being likeliest to do; the properest way to prevent the evil, is to shew them the danger and injustice of it, who are under the greatest temptation to run into it ${ }^{44}$.

Que el peligro principal de la rebelión, es decir, del retorno al estado de guerra proviene de aquellos que están en el poder y no del pueblo se explica, piensa Locke, porque en general "the

Ibid.

lbid.

Ver Ibid., $2^{\circ} \mathrm{T}$ secciones $214-218$.

Ibid., p. 410, 2 T sección 218.

Ibid., p. 416, $2^{\circ}$ T sección 226. 
People, who are more disposed to suffer, than right themselves by Resistance, are not apt to stir"45, y por ende la gente común no se mueve fácilmente en la dirección de la sublevación (a pesar de que en ciertas ocasiones es legítima). En cierto sentido, Locke estaría anticipando la objeción de algunos, ya planteada por Platón, como también por Hobbes, que habían sugerido que la adulación era particularmente efectiva y peligrosa cuando era puesta en ejecución por demagogos que adulan al pueblo, y por ende provocan sedición y facción.

I grant, that the Pride, Ambition, and Turbulency of private Men have sometimes caused great Disorders in Commonwealths, and Factions have been fatal to States and Kingdoms. But whether the mischief hath oftener begun in the Peoples Wantonness, and a Desire to cast off the lawful Authority of their Rulers; or in the Rulers Insolence, and Endeavours to get, and exercise and Arbitrary Power over their People; whether Oppression, or Disobedience gave the first rise to the Disorder, I leave it to impartial History to Determine ${ }^{46}$.

En fin, es de esperar, dice Locke, que sus esfuerzos por refutar, a través de la razón y la argumentación cuidadosa, permitan destinar al olvido los tan perniciosos propósitos de Filmer y sus seguidores, quienes ya han sido dejados al descubierto en tanto aduladores:

This I am sure, their Civil Policy is so new, so dangerous, and so destructive to both Rulers and People, that as former Ages never could bear the broaching of it; so it may be hoped those to come, redeem'd from the Impositions of those Egyptian Under-Taskmasters, will abhor the Memory of such servile Flatterers, who whilst it seem'd to serve their turn, resolv'd all Government into Absolute Tyranny, and would have all Men born to, what their mean Souls fitted them for, Slavery ${ }^{47}$.

\section{CONCLUSIÓN}

En fin, como he dicho, mi propósito en este trabajo ha sido bastante modesto: sólo demostrar que el control de un vicio en particular, la adulación, asociado con otro vicio específico, el orgullo, y con la motivación del beneficio propio en desmedro de los demás, ocupa un lugar importante en la argumentación de Locke y en su defensa de un gobierno de poderes limitados. Los aduladores de hecho forman parte de aquellos "degenerate Men"48 que hacen necesario abandonar el estado natural y configurar la asociación civil y política. Sin embargo, dado que lo político es una frágil construcción humana, se deben establecer claros resguardos a su peligrosa interferencia, incluso en los regímenes más perfectamente ordenados. Los aduladores, letrados serviles e interesados, son un peligro permanente para la vida en libertad, ya que encienden las pasiones más bajas de quienes aspiran al poder, y le atribuyen un espíritu degradado al común de los hombres; un espíritu del que, según Locke, ellos mismos padecen: aquél que está sólo preparado para la sumisión, la obsecuencia y la esclavitud 


\section{REFERENCIAS}

Anderson, Christopher. 1992. "'Safe enough in his honesty and prudence:' The ordinary conduct of government in the thought of John Locke". History of Political Thought, XIII (4): 605-630.

Ashcraft, Richard. 1968. "Locke's State of Nature: Historical Fact or Moral Fiction?". The American Political Science Review, 62 (3): 898-915.

Baier, Annette C. 1995. Moral Prejudices: Essays on Ethics. Cambridge: Harvard University Press.

Berkowitz, Peter. 1999. Virtue and the Making of Modern Liberalism. Princeton: Princeton University Press.

Dunn, John. 1969. The Political Thought of John Locke. Cambridge: Cambridge University Press.

Dunn, John. 1984. "The Concept of Trust in the Politics of John Locke". En Philosophy in History: Essays on the Historiography of Philosophy, editado por Richard Rorty, J.B. Schneewind, y Quentin Skinner. Cambridge: Cambridge University Press, 279-301.

Dunn, John. 1990. "What is Living and What is Dead in the Political Theory of John Locke?" En Interpreting Political Responsibility: Essays 1981-1989. Princeton: Princeton University Press, 9-25.

Dunn, John. 1990. "Trust and Political Agency". En Interpreting Political Responsibility: Essays 1981-1989. Princeton: Princeton University Press, 26-44.

Locke, John. 1991. An Essay Concerning Human Understanding (ed. por P. H. Nidditch). Oxford: Clarendon Press.

Locke, John 1994. Two Treatises of Government (ed. por Peter Laslett). Cambridge: Cambridge University Press.

Locke, John. 2003. Some Thoughs Concerning Education (ed. por John W. y Jean S. Yolton). Oxford: Oxford University Press. Luhmann, Niklas. 1979. Trust and Power, T. Burns y G. Poggi (Eds). Chichester y New York: John Wiley.

Macpherson, Crawford Brough. 1962. The Political Theory of Possessive Individualism: Hobbes to Locke. Oxford: Oxford University Press.

Pateman, Carole. 1988. The Sexual Contract. Stanford: Stanford University Press.

Plutarco. 1985. Obras morales y de costumbres (Moralia). Madrid: Editorial Gredos.

Shakespeare, William. 1977. Julius Caesar, T. S. (Dorsch Ed.). Londres: The Arden Shakespeare.

Shakespeare, William y Middleton, Thomas. 2004. The Life of Timon of Athens. Oxford: Oxford University Press

Starobinski, Jean. 2000. Remedio en el mal: Crítica y legitimación del artificio en la era de las luces (versión original en francés, Éditions Gallimard, 1989) A. Machado Libros, S.A

Stengel, Richard. 2000. You're too Kind: A Brief History of Flattery. New York: Touchstone.

Strauss, Leo. 1950. Natural Right and History. Chicago: The University of Chicago Press.

Sutton, Edward. 1626. The Serpent Anatomized: A Moral Discourse Wherein that FoulSerpentine Vice of Base Creeping Flattery is Manifestly Discovered and Justly Reproved. A needfull Caution for the Credulous, And very usefull for these Times. Kessinger Publishing's Rare Mystical Reprints, s.f.

Teofrasto. 2000. Caracteres, Madrid: Editorial Gredos.

Waldron, Jeremy. 2002. God, Locke and Equality: Christian Foundations in Locke's Political Thought. Cambridge: Cambridge University Press.

Tomás Chuaqui H. es Doctor (Ph. D.) en Ciencia Política, Universidad de Princeton (EE.UU.). Sus áreas de investigación incluyen Teoría Política, Historia del Pensamiento Político y Ética Aplicada. Ha impartido docencia en Chile y Estados Unidos en el área del pensamiento político occidental. Actualmente es Director del Instituto de Ciencia Política de la Pontificia Universidad Católica de Chile.

(E-mail: tchuaqui@puc.cl) 